\title{
Design of Robust Supertwisting Algorithm Based Second-Order Sliding Mode Controller for Nonlinear Systems with Both Matched and Unmatched Uncertainty
}

\author{
Marwa Jouini, Slim Dhahri, and Anis Sellami \\ Laboratory of Engineering of Industrial System and Renewable Energy (LISIER), Higher National Engineering School of Tunis (ENSIT), \\ 5 Avenue Taha Hussein, BP 56, Bab Menara, 1008 Tunis, Tunisia \\ Correspondence should be addressed to Marwa Jouini; marwajouini7@gmail.com
}

Received 4 August 2017; Revised 8 November 2017; Accepted 22 November 2017; Published 13 December 2017

Academic Editor: Olfa Boubaker

Copyright (C) 2017 Marwa Jouini et al. This is an open access article distributed under the Creative Commons Attribution License, which permits unrestricted use, distribution, and reproduction in any medium, provided the original work is properly cited.

\begin{abstract}
This paper proposes a robust supertwisting algorithm (STA) design for nonlinear systems where both matched and unmatched uncertainties are considered. The main contributions reside primarily to conceive a novel structure of STA, in order to ensure the desired performance of the uncertain nonlinear system. The modified algorithm is formed of double closed-loop feedback, in which two linear terms are added to the classical STA. In addition, an integral sliding mode switching surface is proposed to construct the attractiveness and reachability of sliding mode. Sufficient conditions are derived to guarantee the exact differentiation stability in finite time based on Lyapunov function theory. Finally, a comparative study for a variable-length pendulum system illustrates the robustness and the effectiveness of the proposed approach compared to other STA schemes.
\end{abstract}

\section{Introduction}

Sliding Mode Control (SMC) strategy is considered an effective methodology for control uncertain systems. This strategy gives a major objective in control system design to attain stability in the presence of uncertainties [1-5]. The design of the SMC systems mainly consists of two steps: the choice of the sliding mode switching surface and the design of the sliding mode controller. Moreover, SMC has believed significant amount of interest due to several advantages, such that fast convergence, high robustness, and invariance to certain internal system parameter variations and its implementation are easy [5-9]. On the other hand, the worst disadvantage of the SMC methodology is the chattering phenomenon. Thus, to reduce this problem of the chattering effect, numerous techniques are proposed in literature [10-13]; one of them is the supertwisting algorithm (STA) method. The STA has become the prototype of Second-Order Sliding Mode Control (SOSMC) algorithm, which has the capability of system robust stabilization, finite time convergence to the sliding surface, and chattering reduction even in the presence of uncertainties [14-16]. Also, it is able to enforce that the system states converge to the sliding variable $[17,18]$. Nevertheless, the more disadvantage in the supertwisting algorithm is difficulty of designing the gains of the signum function, which leads a very slow convergence and slowly setting time response [14]. In this context, several works have been presented recently proving the stability of the STA using Lyapunov theory and presenting an easy synthesis method of these gains [18]. In addition, great effort has been devoted to enhance the convergence and the robustness of the traditional STA. We can quote some methods: paper [19] proposes the addition of a new term in the classical STA, which leads to improved convergence. In $[18,20]$, the regulation mechanism has been modified by adding a linear term of the sliding variable to the traditional STA. To this end, the aforementioned methods can be only improving the speed convergence of the sliding variable in zero, but they lead a large overshoot of the system response.

In this paper we focus on developing a new modified structure of STA with bounded uncertainty in order to limit the overshoot and shorten the settling time of the system response. This new structure has double closed-loop feedback terms. The first one consists of an outer loop negative 
feedback to accelerate the sliding variable to close to zero and the second feedback is a correction term about an auxiliary variable to reduce the overshoot. Compared with the existing results, the main contributions of this paper are highlighted as follows:

(i) Apply the proposed method for an uncertain nonlinear system, considering two types of uncertainties such as matched and unmatched.

(ii) An integral sliding surface is designed to construct the reachability of the sliding mode.

(iii) A variable-length pendulum system is included to illustrate the applicability of the proposed STA and a comparative study is established with other STA schemes.

The rest of this paper is organized as follows: Section 2 describes the mathematical system description and the problem formulation. The proposed approach is detailed in Section 3. In Section 4, the proving of reaching condition using the Lyapunov function is given. Simulation results are presented in Section 5, and conclusion remarks are in Section 6.

\section{Mathematical System Description and Problem Formulation}

Consider a second-order uncertain nonlinear system described by the state equation:

$$
\begin{aligned}
\dot{x}_{1}(t)= & x_{2}(t), \\
\dot{x}_{2}(t)= & f(t, x)+\Delta f(t, x) \\
& +[g(t, x)+\Delta g(t, x)] u(t), \\
y(t)= & x_{1}(t),
\end{aligned}
$$

where $x_{1}(t), x_{2}(t)$ are the state variables of the system, $x(t)=$ $\left[x_{1}(t), x_{2}(t)\right]^{T} \in \mathbb{R}^{2}$ is the state vector, $u(t) \in \mathbb{R}$ is the input signal control, and $y(t)$ denotes the output vector. $f(t, x)$ and $g(t, x)$ represent the nonlinear dynamic function and the nonlinear control function, respectively. $\Delta f(t, x)$ and $\Delta g(t, x)$ are the corresponding unknown uncertainties of nonlinear vector which can be regarded as satisfying the following assumptions.

Assumption 1. The matched uncertainty $\Delta g(t, x)$ is assumed to be bounded by the unknown scalar $\zeta>0$ such that

$$
\|\Delta g(t, x)\| \leqslant \zeta
$$

Assumption 2. There exists an unknown nonnegative nonlinear function $\gamma(t, x)$ such that the unmatched uncertainty $\Delta f(t, x)$ is bounded as

$$
\|\Delta f(t, x)\| \leqslant \gamma(t, x) .
$$

The main objective of this paper is to design a control input signal for the nonlinear uncertain system (1), which satisfies assumptions (2) and (3), such that the sliding variable $\sigma(t)$ converges to zero in finite time.
Let us consider the classical STA with matched uncertainty used for the design of second-order sliding mode controller $[18,19]$ :

$$
\begin{aligned}
& \dot{\sigma}(t)=-\lambda_{1}|\sigma(t)|^{1 / 2} \operatorname{sign}(\sigma(t))+v(t), \\
& \dot{v}(t)=-\lambda_{3} \operatorname{sign}(\sigma(t))+\Theta(t),
\end{aligned}
$$

where $\sigma(t)$ and $v(t)$ are, respectively, the sliding variable and the auxiliary variable, $\lambda_{1}$ and $\lambda_{3}$ are some positive constants, and the uncertainty $\Theta(t)$ can be expressed as follows:

$$
\Theta(t)=k(t) \operatorname{sign}(\sigma(t)),
$$

where $k(t)$ is the amplitude of uncertainty. Its value should satisfy the following inequalities:

$$
\begin{aligned}
0 & \leqslant k(t) \leqslant M, \\
|\dot{k}(t)| & \leq N,
\end{aligned}
$$

where $M$ and $N$ are positive constants.

In order to accelerate the convergence of the sliding variable $\sigma(t)$ to zero, the gain of signum function $\left(\lambda_{1}, \lambda_{3}\right)$ must have large values. On the other hand, the values of $\lambda_{1}$ and $\lambda_{3}$ should be as small as possible to reduce the chattering phenomenon [19]. To avoid the conflict, many researches $[18,20,21]$ propose the following STA system:

$$
\begin{aligned}
& \dot{\sigma}(t)=-\lambda_{1}|\sigma(t)|^{1 / 2} \operatorname{sign}(\sigma(t))-\lambda_{2} \sigma(t)+v(t) \\
& \dot{v}(t)=-\lambda_{3} \operatorname{sign}(\sigma(t))-\lambda_{4} \sigma(t)+\Theta(t)
\end{aligned}
$$

where $\lambda_{2}$ and $\lambda_{4}$ are positive constants.

The modified structure (7) is used to obtain a faster convergence of $\sigma(t)$. However, if the absolute value of $\dot{v}(t)$ increased, then $v$ increased also; consequently this produces a long setting time and overshoot of sliding variable. To solve this problem, we propose a novel modified STA in the next section.

\section{Proposed Approach}

The objective of this paper is to ameliorate the structure of supertwisting algorithm in order to improve the convergence of sliding variable. However, the proposed method includes a new structure to limit the absolute value of $v(t)$ defined in (7), in which a negative feedback term about $v(t)$ to $\dot{v}(t)$ is added. The new modified algorithm with double closed-loop feedback can be formulated as follows:

$$
\begin{aligned}
& \dot{\sigma}(t)=-\lambda_{1}|\sigma(t)|^{1 / 2} \operatorname{sign}(\sigma(t))-\lambda_{2} \sigma(t)+v(t) \\
& \dot{v}(t)=-\lambda_{3} \operatorname{sign}(\sigma(t))-\lambda_{4} v(t)+\Theta(t),
\end{aligned}
$$

where $\lambda_{1}, \lambda_{2}, \lambda_{3}, \lambda_{4}$ are positive constants. The parameters of the novel modified STA can be selected according to the matched uncertainty (5). $\lambda_{1}$ and $\lambda_{3}$ can be fitted to reduce the chattering phenomenon and $\lambda_{2}$ and $\lambda_{4}$ can be adjusted to guarantee the convergence of the sliding variable $\sigma(t)$. Using relation (8) and the initial conditions $(\sigma(0)>0, v(0)=0$, 
$\Theta(0)=0$ ), we can calculate the regulation mechanism as follows:

$$
v(t)=-\frac{\lambda_{3}}{\lambda_{4}}\left(1-e^{-\lambda_{4} t}\right) .
$$

From (8) and (9), we can note that the overshoot of the sliding variable can be reduced by $-\lambda_{4} v(t)$ and the linear correction term $-\lambda_{2} \sigma(t)$ makes the system faster. Indeed, the performance advantages of the new structure of ST are achieved.

3.1. Stability Analysis of the Novel Structure of STA. In this section, the task is to determinate sufficient conditions to ensure the robustness of the modified algorithm with matched uncertainty (5). Relation (8) can be driven as a mathematical model:

$$
\begin{aligned}
\dot{X}_{1}(t)= & -\lambda_{1}\left|X_{1}(t)\right|^{1 / 2} \operatorname{sign}\left(X_{1}(t)\right)-\lambda_{2} X_{1}(t) \\
& +X_{2}(t), \\
\dot{X}_{2}(t)= & -\lambda_{3} \operatorname{sign}\left(X_{1}(t)\right)-\lambda_{4} X_{2}(t) \\
& +k(t) \operatorname{sign}\left(X_{1}(t)\right),
\end{aligned}
$$

where $\left[\begin{array}{l}\sigma(t) \\ v(t)\end{array}\right]=\left[\begin{array}{l}X_{1}(t) \\ X_{2}(t)\end{array}\right]$.

To facilitate further development, we take $z=\left[\begin{array}{l}z_{1} \\ z_{2}\end{array}\right]=$ $\left[\begin{array}{c}\left|X_{1}(t)\right|^{1 / 2} \operatorname{sign}\left(X_{1}(t)\right) \\ X_{2}(t)\end{array}\right]$.

The derivative of the vector $z$ using (10) is given by

$$
\dot{z}=\frac{1}{\left|z_{1}\right|} A z+\frac{1}{\left|z_{1}\right|}\left[\begin{array}{c}
0 \\
k(t) z_{1}
\end{array}\right]=\frac{1}{\left|z_{1}\right|} Q z,
$$

where

$$
\begin{aligned}
\left|z_{1}\right| & =\left|X_{1}\right|^{1 / 2}, \\
A & =\left[\begin{array}{cc}
-\frac{\lambda_{1}}{2}-\frac{\lambda_{2}}{2}\left|z_{1}\right| & \frac{1}{2} \\
-\lambda_{3} & -\lambda_{4}\left|z_{1}\right|
\end{array}\right], \\
Q & =\left[\begin{array}{cc}
-\frac{\lambda_{1}}{2}-\frac{\lambda_{2}}{2}\left|z_{1}\right| & \frac{1}{2} \\
-\lambda_{3}+k(t) & -\lambda_{4}\left|z_{1}\right|
\end{array}\right] .
\end{aligned}
$$

Using the fact that $\lambda_{1}, \lambda_{2}, \lambda_{3}$, and $\lambda_{4}$ are positive scalars, then the matrix $A$ is Hurwitz.

Choose $\lambda_{1}=a \lambda_{3}$ and $\lambda_{2}=b \lambda_{4}$, where $a>0$ and $b>$ 0 . The stability of the novel modified STA can be designed according to the following theorem.
Theorem 3. Consider the new modified STA (8) with the matched uncertainty (5). If the parameters $\lambda_{1}, \lambda_{2}, \lambda_{3}$, and $\lambda_{4}$ are positive constants, then the sliding mode of system (1) will be built in finite time; that is, the sliding variable will converge to the sliding surface in finite time.

Proof. Propose the following candidate Lyapunov function with respect to the vector $z$ :

$$
V(z)=z^{T} P z,
$$

where $P$ is a symmetric positive definite matrix as

$$
P=\left[\begin{array}{cc}
\frac{\lambda_{4}}{\lambda_{2}}+\left(\frac{2 \lambda_{3}}{\lambda_{1}}\right)^{2} & -\frac{2 \lambda_{3}}{\lambda_{1}} \\
-\frac{2 \lambda_{3}}{\lambda_{1}} & 1
\end{array}\right]=\left[\begin{array}{cc}
b+\left(\frac{2}{a}\right)^{2} & -\frac{2}{a} \\
-\frac{2}{a} & 1
\end{array}\right]
$$

It is easy to show that the Lyapunov function (13) can be bounded from both sides [17] by

$$
\alpha_{\min }\{P\}\|z\|^{2} \leqslant V(z) \leqslant \alpha_{\max }\{P\}\|z\|^{2}
$$

where $\alpha_{\min }$ and $\alpha_{\max }$ are, respectively, the minimum and maximum eigenvalues of $P$ and $\|z\|^{2}$ is the Euclidean norm of $z$.

Using relation (15), it results that

$$
\begin{aligned}
& \|z\|^{2} \geqslant \frac{V(z)}{\alpha_{\max }\{P\}}, \\
& \|z\|^{2} \leqslant \frac{V(z)}{\alpha_{\min }\{P\}} .
\end{aligned}
$$

According to (17), it turns out that

$$
\left|z_{1}\right| \leqslant\|z\| \leqslant \frac{V^{1 / 2}(z)}{\alpha_{\min }^{1 / 2}\{P\}} .
$$

The time derivative of $V(z)$ can be calculated as follows:

$$
\begin{aligned}
\dot{V}(z) & =z^{T} P \dot{z}+\dot{z}^{T} P z=z^{T} P \frac{1}{\left|z_{1}\right|} Q z+\frac{1}{\left|z_{1}\right|} Q^{T} z^{T} P z \\
& =\frac{1}{\left|z_{1}\right|} z^{T}\left[P Q+Q^{T} P\right] z=-z^{T} G z,
\end{aligned}
$$

where

$$
G=\left[\begin{array}{cc}
\left(\frac{4 b}{a^{2}}+b^{2}\right) \lambda_{4}+\frac{a b \lambda_{3}}{\left|z_{1}\right|}+\frac{4 k(t)}{a\left|z_{1}\right|} & -\frac{(2+b) \lambda_{4}}{a}-\frac{2}{a^{2}\left|z_{1}\right|}-\frac{b}{2\left|z_{1}\right|}-\frac{k(t)}{\left|z_{1}\right|} \\
-\frac{(2+b) \lambda_{4}}{a}-\frac{2}{a^{2}\left|z_{1}\right|}-\frac{b}{2\left|z_{1}\right|}-\frac{k(t)}{\left|z_{1}\right|} & 2 \lambda_{4}+\frac{2}{a\left|z_{1}\right|}
\end{array}\right] .
$$


To guarantee $\dot{V}(z)$ is negative definite, (19) can be handled as follows:

$$
\dot{V}(z)=-z^{T} G_{1} z-\frac{1}{\left|z_{1}\right|} z^{T} G_{2} z
$$

with

$$
\begin{aligned}
& G_{1}=\left[\begin{array}{cc}
\left(\frac{4 b}{a^{2}}+b^{2}\right) \lambda_{4} & -\frac{(2+b) \lambda_{4}}{a} \\
-\frac{(2+b) \lambda_{4}}{a} & 2 \lambda_{4}
\end{array}\right], \\
& G_{2}=\left[\begin{array}{cc}
\left(a b \lambda_{3}+\frac{4 k(t)}{a}\right) & -\left(\frac{2}{a^{2}}+\frac{b}{2}+k(t)\right) \\
-\left(\frac{2}{a^{2}}+\frac{b}{2}+k(t)\right) & \frac{2}{a}
\end{array}\right],
\end{aligned}
$$

where $G_{1}$ and $G_{2}$ are symmetrical matrices.

The time derivative of $V(z)$ is bounded as follows:

$$
\begin{aligned}
\dot{V}(z) & \leqslant-\alpha_{\min }\left\{G_{1}\right\}\|z\|^{2}-\frac{1}{\left|z_{1}\right|} \alpha_{\min }\left\{G_{2}\right\}\|z\|^{2} \\
& \leqslant-\left(\alpha_{\min }\left\{G_{1}\right\}+\frac{1}{\left|z_{1}\right|} \alpha_{\min }\left\{G_{2}\right\}\right)\|z\|^{2},
\end{aligned}
$$

where $\alpha_{\min }\left(\left\{G_{1}\right\},\left\{G_{2}\right\}\right)$ are, respectively, the minimum eigenvalue of $G_{1}$ and $G_{2}$. follows:

Using (16)-(18), the inequality (23) can be expressed as

$$
\begin{aligned}
\dot{V}(z) \leqslant & -\underbrace{\frac{\alpha_{\min }\left\{G_{1}\right\}}{\alpha_{\max }\{P\}}}_{=\beta_{1}} V(z) \\
& -\underbrace{\frac{\alpha_{\min }{ }^{1 / 2}\{P\} \alpha_{\min }\left\{G_{2}\right\}}{\alpha_{\max }\{P\}}}_{=\beta_{2}} V^{1 / 2}(z),
\end{aligned}
$$

where $\beta_{1}$ and $\beta_{2}$ are positive constants.

Hence, the time derivative of $V(z)$ is bounded as follows:

$$
\dot{V}(z) \leqslant-\beta_{1} V(z)-\beta_{2} V^{1 / 2}(z) .
$$

Equation (25) shows that the derivative of the Lyapunov function is negative definite $(V(z) \leqslant 0)$. We can conclude that the new modified STA (8) with matched uncertainty (5) converges to zero in finite time.

3.2. Comparative Study. A comparative analysis has been made to compare the convergence performances between the different structures of supertwisting algorithm: the classical STA, the modified STA (MSTA) (see references [18-21]), and the novel modified STA. In order to obtain smaller amplitude chattering, the parameters $\lambda_{1}$ and $\lambda_{3}$ should be adjusted as small as possible, and the parameters $\lambda_{2}$ and $\lambda_{4}$ are selected to guarantee the convergence of the sliding variable. These parameters are chosen as $\lambda_{1}=0.5, \lambda_{2}=2, \lambda_{3}=0.6$, and
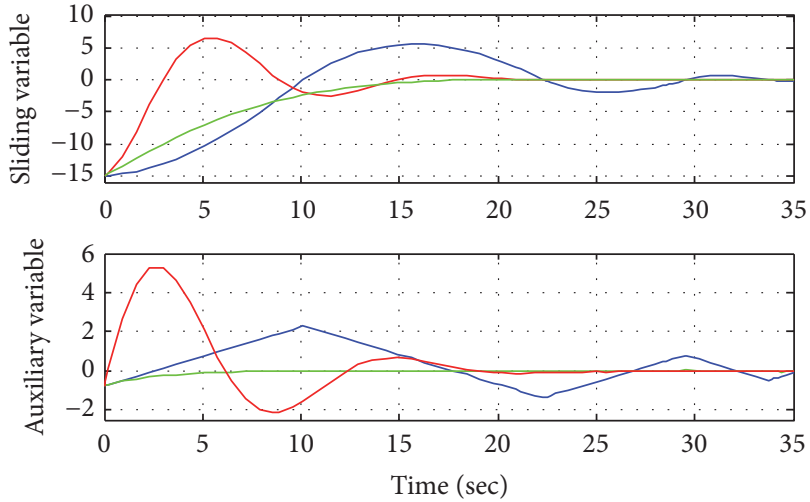

FIGURE 1: Dynamic of the sliding variable and the auxiliary variable with different structures of STA (blue line: the classical STA, red line: the modified STA, and green line the novel modified STA).

TABLE 1: The performances of the different structures of STA.

\begin{tabular}{lcc}
\hline Structure & Overshoot & Settling time \\
\hline Classical STA & $5.5 \%$ & $33.8 \mathrm{~s}$ \\
Modified STA & $6.52 \%$ & $19 \mathrm{~s}$ \\
Novel modified STA & $1.1 \%$ & $18 \mathrm{~s}$ \\
\hline
\end{tabular}

$\lambda_{4}=2$. Figure 1 shows the dynamic of the sliding variable and the auxiliary variable with the following initial conditions: $\sigma(0)=-15$ and $v(0)=-0.75$.

It can be observed in Figure 1 and Table 1 that a faster speed of the sliding variable approaching zero is obtained in modified STA and the novel modified STA, as they add the same linear correction term $-\lambda_{2} \sigma(t)$ compared to classical STA. Moreover, by the effect of the linear feedback term $-\lambda_{4} v(t)$ added in the novel modified structure, the sliding variable $\sigma(t)$ obtains small overshoot (1.1\%). Thus, the new structure of STA proposed achieves the best convergence performances in terms of settling time and overshoot.

\section{Sliding Mode Reachability}

To reduce the steady error, an integral term of tracking error is introduced, which makes up the integral sliding surface [22, 23] as follows:

$$
\sigma(t)=\dot{e}(t)+k_{1} e(t)+k_{2} \int_{0}^{t} e(\tau) d \tau,
$$

where $e(t)=y(t)-y_{\text {ref }}(t)=x_{1}(t)-x_{\text {ref }}(t)$ is the tracking error, $y_{\text {ref }}(t)$ denotes the reference trajectory, and $k_{1}$ and $k_{2}$ are positive constants.

The time derivative of (26) yields that

$$
\begin{aligned}
\dot{\sigma}(t)= & \ddot{e}(t)+k_{1} \dot{e}(t)+k_{2} e(t) \\
= & \left(\dot{x}_{2}(t)-\ddot{x}_{\text {ref }}(t)\right)+k_{1}\left(x_{2}(t)-\dot{x}_{\text {ref }}(t)\right) \\
& +k_{2}\left(x_{1}(t)-x_{\text {ref }}(t)\right) .
\end{aligned}
$$


Using (1), the above equation can be rewritten as

$$
\begin{aligned}
\dot{\sigma}(t) & =(f(t, x)+\Delta f(t, x) \\
+ & \left.(g(t, x)+\Delta g(t, x)) u(t)-\ddot{x}_{\text {ref }}(t)\right)+k_{1}\left(x_{2}(t)\right. \\
- & \left.\dot{x}_{\text {ref }}(t)\right)+k_{2}\left(x_{1}(t)-x_{\text {ref }}(t)\right) .
\end{aligned}
$$

Substituting (8) into (28), the control law is given by

$$
\begin{aligned}
u(t) & =(g(t, x)+\Delta g(t, x))^{-1}[-f(t, x)-\Delta f(t, x) \\
& +\ddot{x}_{\text {ref }}(t)-k_{1}\left(x_{2}(t)-\dot{x}_{\text {ref }}(t)\right) \\
& -k_{2}\left(x_{1}(t)-x_{\text {ref }}(t)\right)-\lambda_{1}|\sigma(t)|^{1 / 2} \operatorname{sign}(\sigma(t)) \\
& \left.-\lambda_{2} \sigma(t)+v(t)\right] .
\end{aligned}
$$

Theorem 4. Consider a dynamic uncertain second-order system (1) subject both matched and unmatched uncertainties. If the sliding mode surface is selected as (26) and the control input is designed as (29); then, the states variables converge to the trajectories signal and the sliding variable will reach the sliding surface $\sigma(t)=0$ in finite time.

Proof. Consider the Lyapunov function as follows:

$$
V(\sigma, t)=\frac{1}{2} \sigma(t)^{2} .
$$

To ensure that the sliding mode is reached in finite time, the derivative of $V(\sigma, t)$ with respect to time must be negative definite.

Take the time derivative of Lyapunov function $V(\sigma, t)$ as follows:

$$
\dot{V}(\sigma, t)=\sigma(t) \dot{\sigma}(t) .
$$

Substituting (28) into (31), we can obtain

$$
\begin{aligned}
& \dot{V}(\sigma, t)=\sigma(t)[(f(t, x)+\Delta f(t, x) \\
& \left.\quad+(g(t, x)+\Delta g(t, x)) u(t)-\ddot{x}_{\text {ref }}(t)\right)+k_{1}\left(x_{2}(t)\right. \\
& \left.\left.\quad-\dot{x}_{\text {ref }}(t)\right)+k_{2}\left(x_{1}(t)-x_{\text {ref }}(t)\right)\right] .
\end{aligned}
$$

Substituting equation (29) into (32), it results that

$$
\begin{aligned}
& \dot{V}(\sigma, t)=\sigma(t)\left[(g(t, x)+\Delta g(t, x))^{-1}\right. \\
& \left.\quad \cdot\left(-\lambda_{1}|\sigma(t)|^{1 / 2} \operatorname{sign}(\sigma(t))-\lambda_{2} \sigma(t)+v(t)\right)\right] .
\end{aligned}
$$

Using (2) and (9), (33) can be calculated as

$$
\begin{aligned}
& \dot{V}(\sigma, t) \leqslant-\left[(|g(t, x)|+\zeta)^{-1}\right]\left[\left(\lambda_{1}|\sigma(t)|^{3 / 2}\right.\right. \\
& \left.\left.+\lambda_{2}|\sigma(t)|^{2}-|\sigma(t)| \frac{\lambda_{3}}{\lambda_{4}}\left(1-e^{-\lambda_{4} t}\right)\right)\right] \leqslant-|\sigma(t)| \\
& \quad \cdot(|g(t, x)|+\zeta)^{-1}\left[\lambda_{1}|\sigma(t)|^{1 / 2}+\lambda_{2}|\sigma(t)|+\frac{\lambda_{3}}{\lambda_{4}}(1\right. \\
& \left.\left.\quad-e^{-\lambda_{4} t}\right)\right]
\end{aligned}
$$

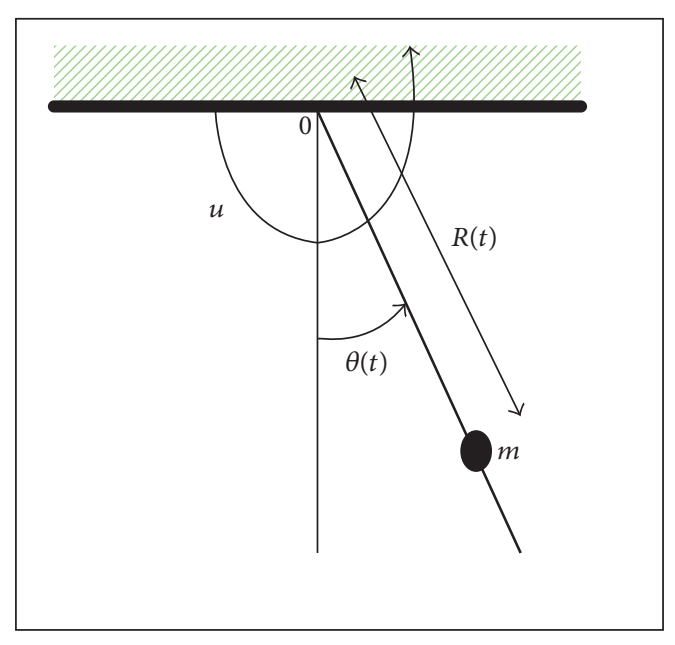

Figure 2: Pendulum system.

TABLE 2: Parameters values of the pendulum system.

\begin{tabular}{lcc}
\hline Notations & Significance & Values \\
\hline$m$ & The mass & $1 \mathrm{~kg}$ \\
$g$ & The acceleration gravity & $9.81 \mathrm{~m} / \mathrm{s}^{2}$ \\
$R$ & The distance from & $0.8+0.1 \sin 8 t+0.3 \cos 4 t$ \\
$\theta_{\text {ref }}$ & $\quad$ The reference trajectory & $0.5 \sin 0.5 t+0.5 \cos t$ \\
\hline
\end{tabular}

and then $\dot{V}(\sigma, t)$ can be rewritten as

$$
\dot{V}(\sigma, t) \leqslant-\eta|\sigma(t)|,
$$

where $\eta=(|g(t, x)|+\zeta)^{-1}\left(\lambda_{1}|\sigma(t)|^{1 / 2}+\lambda_{2}|\sigma(t)|+\left(\lambda_{3} / \lambda_{4}\right)(1-\right.$ $\left.e^{-\lambda_{4} t}\right)$ ). It is clear from (35) that if $\eta>0$, then $\dot{V}(\sigma, t)$ is negative definite.

Therefore, we conclude that the sliding mode can be reached in finite time $\sigma(t)=0$ and the control law as defined in (29) would guarantee that $x(t) \rightarrow x_{\text {ref }}(t)$ when $t \rightarrow \infty$.

\section{Simulation Results and Discussions}

In this section, the effectiveness of our developed algorithm will be illustrated using a pendulum system example. We compare our results with those obtained by other existing methods such as classical and modified STA (MSTA). The pendulum system is driven by an engine installed on the top side, which is called control torque $u$ (see Figure 2) [24]. Thus, the task is tracking some function $\theta_{\text {ref }}(t)$ in real time by the oscillation angle $\theta(t)$ of the road.

The dynamic equation of the pendulum system is expressed by

$$
\ddot{\theta}(t)=-2 \frac{\dot{R}}{R} \dot{\theta}(t)-\frac{g}{R} \sin (\theta(t))+\frac{1}{m R^{2}} u(t) .
$$

The parameters of this system are presented in Table 2. 


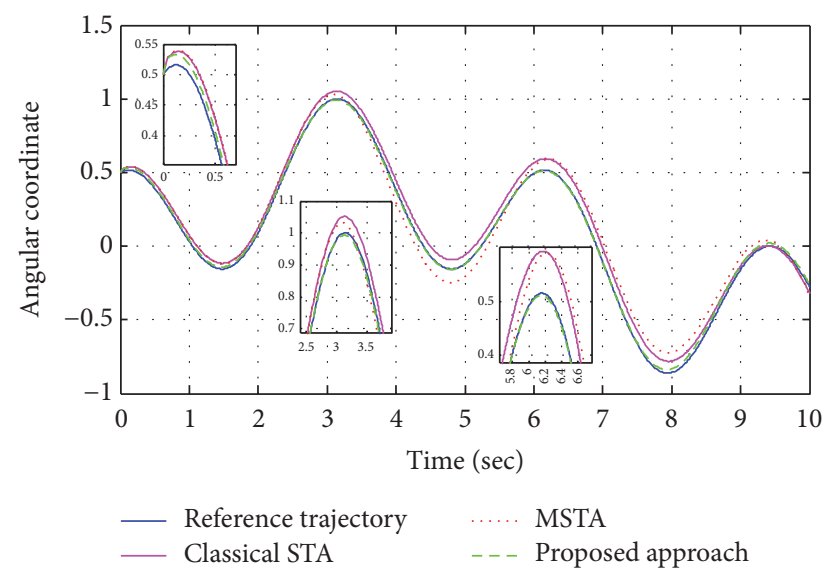

FIgURE 3: The response $x_{1}(t)$ of the system with matched and unmatched uncertainties.

We consider $x_{1}(t)=\theta(t)$ and $x_{2}(t)=\dot{\theta}(t)$; the state space of the pendulum system can be described by

$$
\begin{aligned}
\dot{x}_{1}(t)= & x_{2}(t), \\
\dot{x}_{2}(t)= & -2 \frac{\dot{R}}{R} x_{2}(t)-\frac{g}{R} \sin \left(x_{1}(t)\right)+\Delta f(t, x) \\
& +\left(\frac{1}{m R^{2}}+\Delta g(t, x)\right) u(t), \\
y(t)= & x_{1}(t) .
\end{aligned}
$$

The unmatched and matched uncertainties are chosen by the following equations:

$$
\begin{aligned}
\Delta f(x, t)= & 0.1 \cos 3 t^{2}+x_{1}^{2}(t) \sin \left(0.5 x_{1}(t)\right) \\
& +0.2 \cos \left(2 x_{2}^{2}(t)\right)+x_{2}^{2}(t), \\
\Delta g(t, x)= & 0.1 \cos \left(2 x_{1}(t)\right) .
\end{aligned}
$$

According to Assumptions 1 and 2, it can be verified that $\gamma(t, x)=0.3+\|x\|^{2}$ and $\zeta=0.1$. The bounded uncertainty of the supertwisting algorithm can be selected as $\Theta(t)=$ $(0.1 \sin (2 \pi t)) \operatorname{sign}(\sigma(t))$.

In the simulation, the initial values for the state system (37) are selected as $x_{1}(0)=0.5$ and $x_{2}(0)=1$ and the following parameters are used as $k_{1}=0.2, k_{2}=0.01, \lambda_{1}=$ $25, \lambda_{2}=8, \lambda_{3}=2$, and $\lambda_{4}=1$.

Figures 3 and 4 show, respectively, the system state variables $x_{1}(t)$ and $x_{2}(t)$ of the pendulum system with both matched and unmatched uncertainties using different approaches. It is easy to see that the angular coordinate and the angular velocity of the system for the three methods converge to desired trajectories without chattering phenomenon despite the presence of uncertainties. For comparison, we can note that the performance results of our proposed method are better than what is reported in [18] and [21].

However, the results of Figures 5 and 6 show that the proposed structure of STA outperform the MSTA. Indeed,

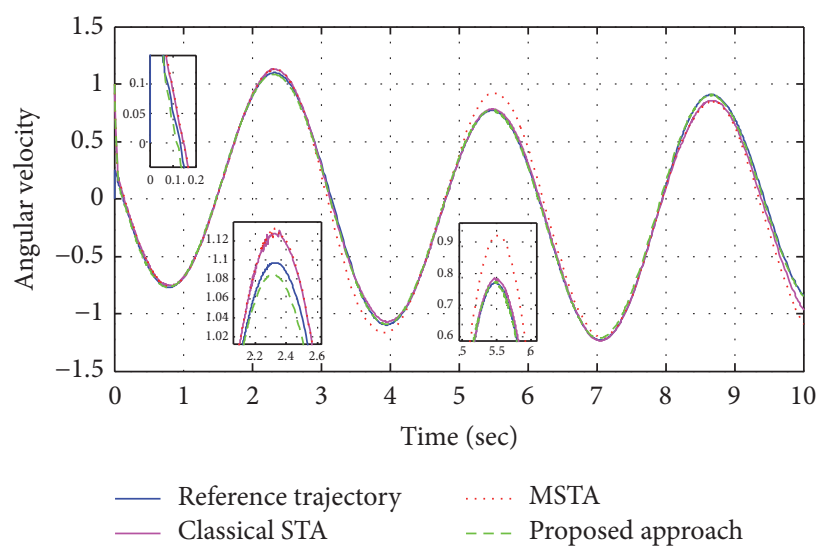

Figure 4: The response $x_{2}(t)$ of the system with matched and unmatched uncertainties.

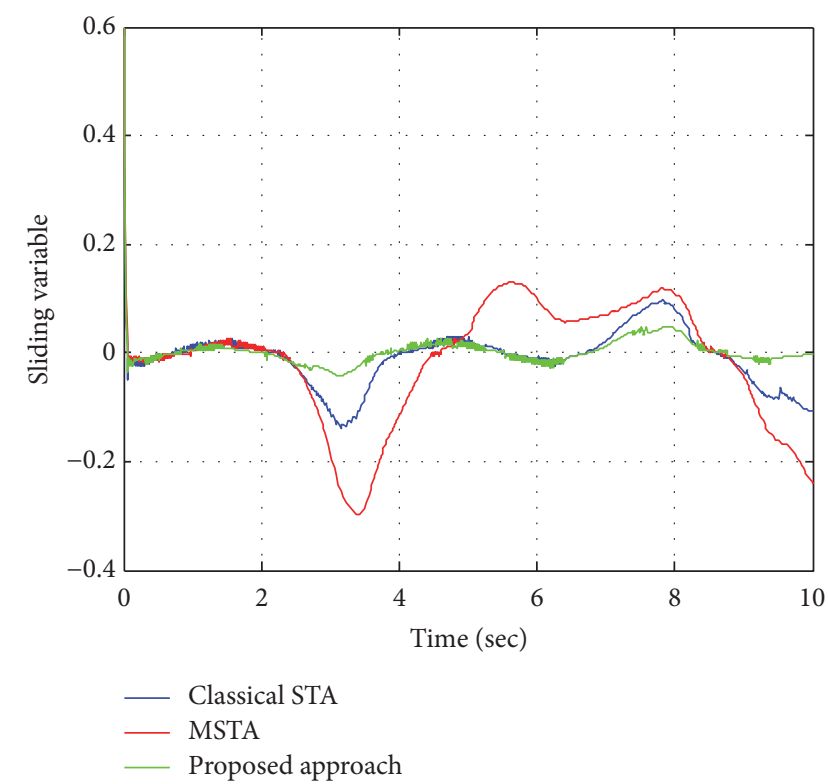

FIGURE 5: Sliding variable $\sigma(t)$.

Figure 5 illustrates the evolution responses of the sliding variable for the different structures of supertwisting algorithm. We can note the novel modified STA given a faster convergence of sliding variable and smallest tracking error (Figure 6) compared to other results obtained by especially using the classical and modified STA [18-21]. A similar analysis can be seen in Table 3 . The performance indexes of different methods listed in this table confirm that the novel modified STA has a faster settling time and shorting overshoot. This indicates that the proposed approach is more resistant to uncertainties.

The time evolution of the control signals shown in Figure 7 clearly demonstrates that the case of using the novel structure is producing small vibrations. In addition, the superiority of the new modified STA is shown in Table 4. Consequently, the proposed control method has good performance qualities for the nonlinear model of pendulum 


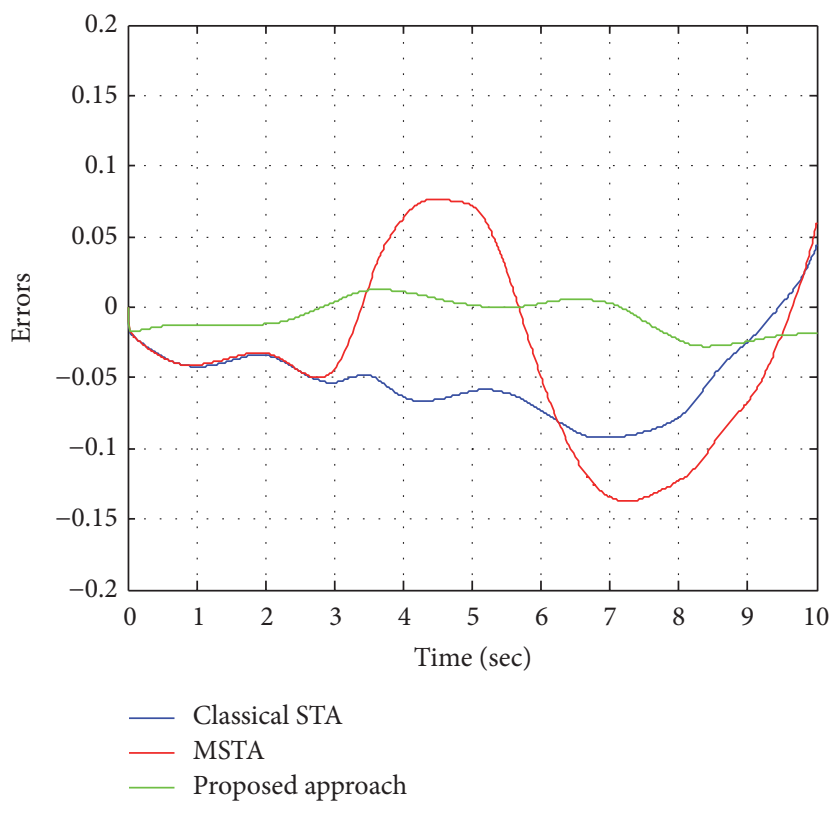

FIGURE 6: Tracking error $e(t)$.

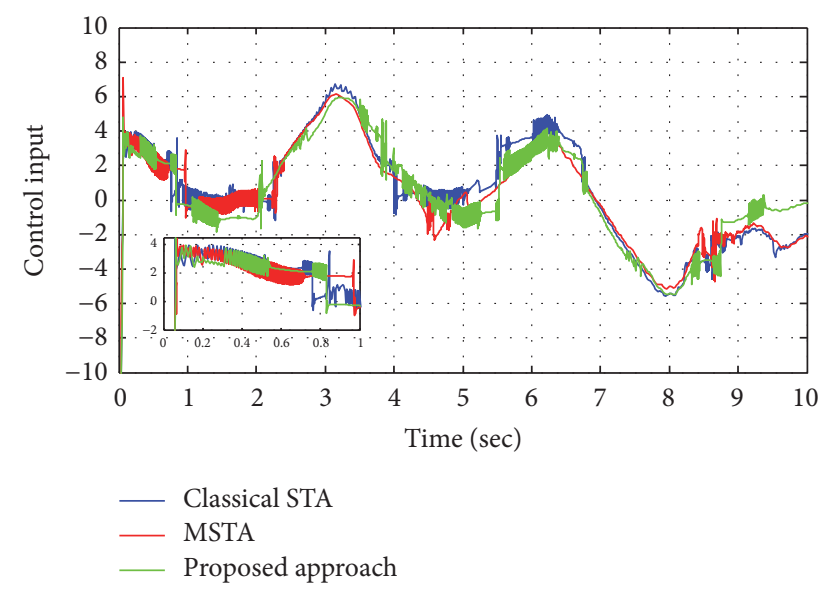

FIgURE 7: Control inputs $u(t)$ for pendulum system.

TABLE 3: Comparison performances of the super-twisting algorithm.

\begin{tabular}{lcc}
\hline $\begin{array}{l}\text { Supertwisting } \\
\text { algorithm }\end{array}$ & Overshoot & Settling time \\
\hline \multirow{3}{*}{ Classical [18] } & $D_{\sigma}=1.4 \%$ & $t_{r, \sigma}=0.8 \mathrm{sec}$ \\
& $D_{x_{1}}=10 \%$ & $t_{r, x_{1}}=2 \mathrm{sec}$ \\
& $D_{x_{2}}=30 \%$ & $t_{r, x_{2}}=0.18 \mathrm{sec}$ \\
\hline Modified & $D_{\sigma}=1.12 \%$ & $t_{r, \sigma}=0.78 \mathrm{sec}$ \\
{$[18-21]$} & $D_{x_{1}}=6 \%$ & $t_{r, x_{1}}=0.8 \mathrm{sec}$ \\
& $D_{x_{2}}=26 \%$ & $t_{r, x_{2}}=0.18 \mathrm{sec}$ \\
\hline \multirow{2}{*}{ Proposed } & $D_{\sigma}=0.8 \%$ & $t_{r, \sigma}=0.75 \mathrm{sec}$ \\
approach & $D_{x_{1}}=2 \%$ & $t_{r, x_{1}}=0.5 \mathrm{sec}$ \\
& $D_{x_{2}}=10 \%$ & $t_{r, x_{2}}=0.15 \mathrm{sec}$ \\
\hline
\end{tabular}

system in the presence of matched and unmatched uncertainties.
TABLE 4: Control effort measures for different structures of STA.

\begin{tabular}{lccc}
\hline $\begin{array}{l}\text { Control input } \\
\text { under }\end{array}$ & Classical STA & $\begin{array}{c}\text { Modified } \\
\text { STA }\end{array}$ & $\begin{array}{c}\text { Novel modified } \\
\text { STA }\end{array}$ \\
\hline$\|u\|$ & 26.41 & 26.53 & 26.00 \\
\hline
\end{tabular}

\section{Conclusion}

In this paper, a new structure of supertwisting algorithm is proposed and applied to an uncertain nonlinear system subject to matched and unmatched uncertainties. The main idea consists of adding two closed-loop feedback terms to the traditional supertwisting with bounded uncertainty in order to ameliorate the performances of the system response. Theoretical analysis is achieved to guarantee the stability using the Lyapunov function. Therefore, a comparative study demonstrates that the proposed approach can improve the convergence of the sliding variable and makes the system faster despite the presence of uncertainties. The proposed approach shows favorable results compared with the methods reported in the literature.

\section{Conflicts of Interest}

There are no any conflicts of interest related to this paper.

\section{References}

[1] A. Levant, "Sliding order and sliding accuracy in sliding mode control," International Journal of Control, vol. 58, no. 6, pp. 12471263, 1993.

[2] X. Zhang, "Integral sliding mode control for non-linear systems with mismatched uncertainty based on quadratic sliding mode," The Journal of Engineering, p. 6, 2015.

[3] J. Huang, Z.-H. Guan, T. Matsuno, T. Fukuda, and K. Sekiyama, "Sliding-mode velocity control of mobile-wheeled invertedpendulum systems," IEEE Transactions on Robotics, vol. 26, no. 4, pp. 750-758, 2010.

[4] F. S. Ahmed, S. Laghrouche, and M. El Bagdouri, "Second-order sliding mode based output-feedback control of an engine air path actuator in presence of uncertainties," in Proceedings of the 1 st Conference on Control and Fault-Tolerant Systems (SysTol '10), pp. 50-56, Nice, France, October 2010.

[5] Y. Niu, Z. Wang, and X. Wang, "Robust sliding mode design for uncertain stochastic systems based on $\mathrm{H}_{\infty}$ control method," Optimal Control Applications and Methods, vol. 31, no. 2, pp. 93104,2010

[6] A. H. Alqahtani and V. I. Utkin, "Self-optimization of photovoltaic system power generation based on sliding mode control," in Proceedings of the 38th Annual Conference on IEEE Industrial Electronics Society (IECON '12), pp. 3468-3474, IEEE, Montreal, Canada, October 2012.

[7] H. Fukushima, K. Muro, and F. Matsuno, "Sliding-mode control for transformation to an inverted pendulum mode of a mobile robot with wheel-arms," IEEE Transactions on Industrial Electronics, vol. 62, no. 7, pp. 4257-4266, 2015.

[8] S. Wajdi, S. Anis, and G. Garcia, "Robust sliding mode control approach for systems affected by unmatched uncertainties using $\mathrm{H}_{\infty}$ with pole clustering constraints," Optimal Control Applications and Methods, vol. 36, no. 6, pp. 919-935, 2015. 
[9] K. Xing, J. Huang, J. He, Y. Wang, Q. Xu, and J. Wu, "Sliding mode tracking for actuators comprising pneumatic muscle and torsion spring," Transactions of the Institute of Measurement and Control, vol. 34, no. 2-3, pp. 255-277, 2012.

[10] Y. Feng, F. Han, and X. Yu, "Chattering free full-order slidingmode control," Automatica, vol. 50, no. 4, pp. 1310-1314, 2014.

[11] J. X. Xu, Z. Q. Guo, and T. H. Lee, "Design and implementation of integral sliding-mode control on an underactuated two-wheeled mobile robot," IEEE Transactions on Industrial Electronics, vol. 61, no. 7, pp. 3671-3681, 2014.

[12] H. Lee and V. I. Utkin, "Chattering suppression methods in sliding mode control systems," Annual Reviews in Control, vol. 31, no. 2, pp. 179-188, 2007.

[13] S. Kamal, A. Chalanga, J. Moreno, L. Fridman, and B. Bandyopadhyay, "Higher order super-twisting algorithm," in Proceedings of the 13th International Workshop on Variable Structure Systems (VSS), pp. 1-5, Nantes, France, June 2014.

[14] S. Kuntanapreeda, "Super-twisting sliding-mode traction control of vehicles with tractive force observer," Control Engineering Practice, vol. 38, pp. 26-36, 2015.

[15] M. Jouini, S. Dhahri, N. Amara, and A. Sellami, "Second order and classical sliding mode control of a MAXPID system: a comparison performance," in Proceedings of the 15th International Conference on Sciences and Techniques of Automatic Control and Computer Engineering (STA '14), pp. 936-941, Hammamet, Tunisia, December 2014.

[16] L. Derafa, A. Benallegue, and L. Fridman, "Super twisting control algorithm for the attitude tracking of a four rotors UAV," Journal of The Franklin Institute, vol. 349, no. 2, pp. 685-699, 2012.

[17] J. A. Moreno and M. Osorio, "Strict Lyapunov functions for the super-twisting algorithm," IEEE Transactions on Automatic Control, vol. 57, no. 4, pp. 1035-1040, 2012.

[18] J. A. Moreno, "On strict Lyapunov functions for some non-homogeneous super-twisting algorithms," Journal of The Franklin Institute, vol. 351, no. 4, pp. 1902-1919, 2014.

[19] V. I. Utkin and A. S. Poznyak, "Adaptive sliding mode control with application to super-twist algorithm: equivalent control method," Automatica, vol. 49, no. 1, pp. 39-47, 2013.

[20] J. A. Moreno and M. Osorio, "A Lyapunov approach to secondorder sliding mode controllers and observers," in Proceedings of the 47th IEEE Conference on Decision and Control (CDC '08), pp. 2856-2861, Cancun, Mexico, December 2008.

[21] I. Nagesh and C. Edwards, "A multivariable super-twisting sliding mode approach," Automatica, vol. 50, no. 3, pp. 984-988, 2014.

[22] D. Liu, W. Guo, and W. Wang, "Second-order sliding mode tracking control for the piezoelectric actuator with hysteretic nonlinearity," Journal of Mechanical Science and Technology, vol. 27, no. 1, pp. 199-205, 2013.

[23] X. Zheng, X. Jian, D. Wenzheng, and C. Hongjie, "Nonlinear integral sliding mode control for a second order nonlinear system," Journal of Control Science and Engineering, vol. 2015, Article ID 218198, pp. 1-7, 2015.

[24] A. Levant, "Principles of 2-sliding mode design," Automatica, vol. 43, no. 4, pp. 576-586, 2007. 


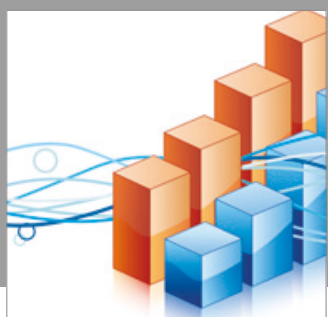

Advances in

Operations Research

vatersals

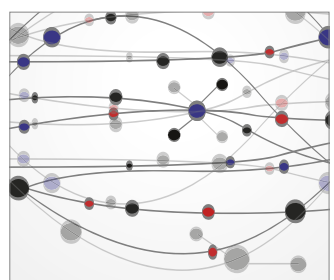

\section{The Scientific} World Journal
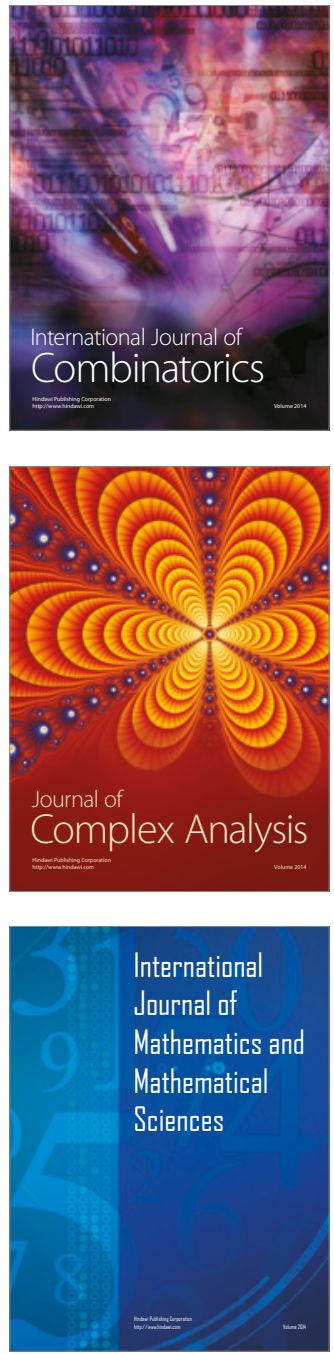
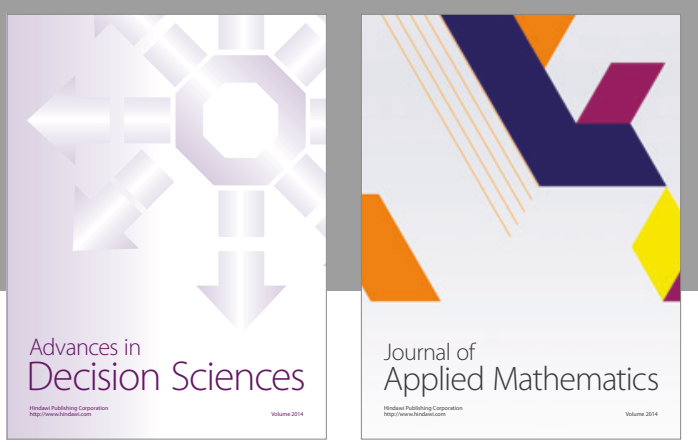

Algebra

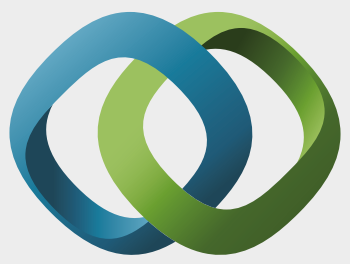

\section{Hindawi}

Submit your manuscripts at

https://www.hindawi.com
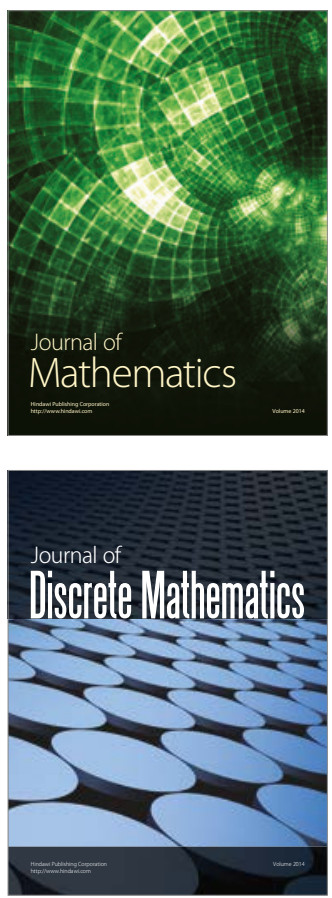

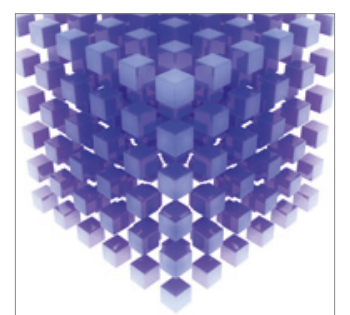

Mathematical Problems in Engineering
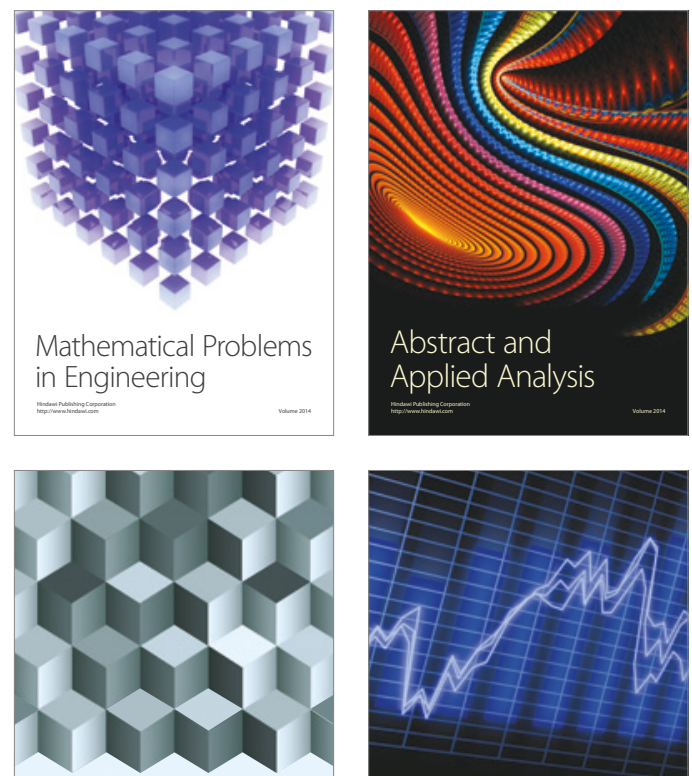

Journal of

Function Spaces

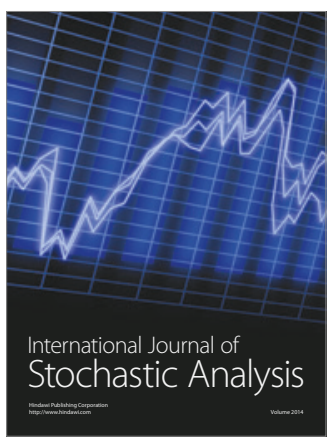

Probability and Statistics
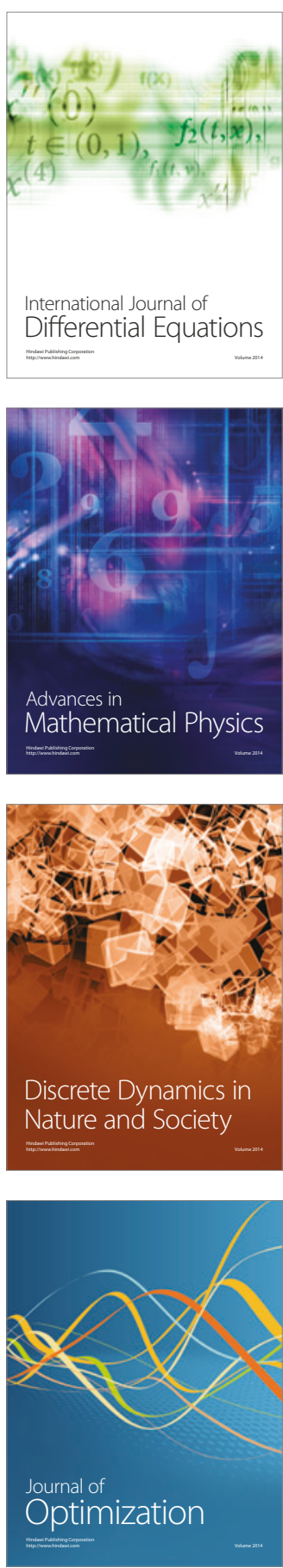